\title{
A Quantile Approach to Integration with Respect to Non-additive Measures
}

\author{
Marta Cardin \\ Department of Economics, \\ University Cà Foscari of Venice \\ Cannaregio 873, 30121Venice - Italy \\ mcardin@unive.it
}

\begin{abstract}
The aim of this paper is to introduce some classes of aggregation functionals when the evaluation scale is a complete lattice. We focus on the notion of quantile of a lattice-valued function which have several properties of its real-valued counterpart and we study a class of aggregation functionals that generalizes Sugeno integrals to the setting of complete lattices. Then we introduce in the real-valued case some classes of aggregation functionals that extend Choquet and Sugeno integrals by considering a multiple quantile model generalizing the approach proposed in [3].
\end{abstract}

Keywords: Completely distributive lattice, quantile, Sugeno integral, Choquet integral.

\section{Introduction}

Aggregation operators are an important mathematical tool for the combination of several inputs in a single outcome that is used in many applied fields and in particular in the area of artificial intelligence for decision making(see [9] for a general background). Real-valued non-additive measures and their associated integrals are widely used aggregation operators. There are many situations where inputs to be aggregated are qualitative and numerical values are used by convenience. Moreover sometimes we need to evaluate objects with a scale that is not totally ordered. As the aim of this paper is to generalize some well known aggregation functionals in a purely ordinal context. In this case only maximum and minimum are used for aggregation of different inputs. So we study aggregation functionals based on a complete lattices and we consider in particular the class of completely distributive lattices. The quantile is a generalization of the concept of median and it play an important role in statistical and economic literature. We study quantile in a ordinal framework and and we consider an axiomatic representation of quantiles as in [6] and [5].

The structure of the paper is as follows. To make this work self-contained in Section 2 we briefly mention some basic concepts on lattices theory and we provide the necessary definitions. Section 3 is devoted to lattice-valued measures and lattice-valued integrals. Finally in Section 4 we introduce some classes of generalized integrals based on a multiple quantiles model. 


\section{Notations and definitions}

To introduce our general framework we will need some algebraic preliminaries. Much of this terminology is well known and for further background in lattice theory we refer the reader to Davey and Priestley [7] or Grätzer [10].

A lattice is an algebraic structure $\langle L ; \wedge, \vee\rangle$ where $L$ is a nonempty set, called universe, and where $\wedge$ and $\vee$ are two binary operations, called meet and join, respectively, which satisfy the following axioms:

(i) (idempotency) for every $a \in L, a \vee a=a \wedge a=a$;

(ii) (commutativity) for every $a, b \in L, a \vee b=b \vee a$ and $a \wedge b=b \wedge a$;

(iii) (associativity) for every $a, b, c \in L, a \vee(b \vee c)=(a \vee b) \vee c$ and $a \wedge(b \wedge c)=$ $(a \wedge b) \wedge c$

(iv) (absorption): for every $a, b \in L, a \wedge(a \vee b)=a$ and $a \vee(a \wedge b)=a$.

Every lattice $L$ constitutes a partially ordered set endowed with the partial order $\leq$ such that for every $x, y \in L$, write $x \leqslant y$ if $x \wedge y=x$ or, equivalently, if $x \vee y=y$. If for every $a, b \in L$, we have $a \leqslant b$ or $b \leqslant a$, then $L$ is said to be a chain. A lattice $L$ is said to be bounded if it has a least and a greatest element, denoted by 0 and 1 , respectively.

A lattice $L$ is said to be distributive, if for every $a, b, c \in L$,

$a \vee(b \wedge c)=(a \vee b) \wedge(a \vee c)$ or, equivalently, $a \wedge(b \vee c)=(a \wedge b) \vee(a \wedge c)$

Clearly, every chain is distributive. A lattice $L$ is said to be complete if for every $S \subseteq L$, its supremum $\bigwedge S:=\bigwedge_{x \in S} x$ and infimum $\bigvee S:=\bigvee_{x \in S} x$ exist. Clearly, every complete lattice is necessarily bounded.

A complete lattice $L$ is said to be completely distributive is the following more stringent distributive law holds

$$
\bigwedge_{i \in I}\left(\bigvee_{j \in J} x_{i j}\right)=\bigvee_{f \in J^{I}}\left(\bigwedge_{i \in I} x_{i f(i)}\right)
$$

for every doubly indexed subset $\left\{x_{i j}: i \in I, j \in J\right\}$ of $L$. Note that every complete chain (in particular, the extended real line and each product of complete chains) is completely distributive. Moreover, complete distributivity reduces to distributivity in the case of finite lattices. Throughout this paper, $A$ denotes an arbitrary nonempty set and $L$ a lattice. The set $L^{A}$ of all functions from $A$ to $L$ constitutes a lattice under the operations $\wedge$ and $\vee$ defined pointwise, i.e.,

$(f \wedge g)(x)=f(x) \wedge g(x) \quad$ and $\quad(f \vee g)(x)=f(x) \vee g(x) \quad$ for every $f, g \in L^{A}$.

In particular, for any lattice $L$, the cartesian product $L^{n}$ also constitutes a lattice by defining the lattice operations componentwise. Observe that if $L$ is bounded (distributive), then $L^{A}$ is also bounded (resp. distributive). We denote by $\mathbf{0}$ and 1 the least and the greatest elements, respectively, of $L^{A}$. Likewise, for each $c \in L$, we denote by $\mathbf{c}$ the constant c map in $L^{A}$. Moreover or each $X \subset A$, 
we denote by $\mathbf{X}$ the characteristic function of $X$ in $L^{A}$ defined by $\mathbf{X}(x)=1$ if $x \in X$ and $\mathbf{X}(x)=0$ if $x \notin X$.

The following notion extends that of homomorphism between lattices. A map $\gamma: L \rightarrow L$, where $L$ is a complete lattice, is said to be continuous if it preserves arbitrary meets and and arbitrary joins, i.e., for every $S \subseteq L$,

$$
\gamma(\bigwedge S)=\bigwedge \gamma(S) \text { and } \gamma(\bigvee S)=\bigvee \gamma(S)
$$

The term continuous is justified by the following fact (see [11]): if $\gamma: L \rightarrow L$ is continuous, then it is continuous with respect to the Lawson topology on $L$.

\section{Lattice-valued measures and lattice-valued integrals}

The following definitions are natural extensions of the well known concepts of real -valued non-additive (or fuzzy) measures and their associated integrals.

We follow the approach proposed by Greco in [13] and more recently by Ban and Fechete in [2] for lattice-valued measures and integrals and we refer to [16] and [17] for the standard case. Let $(A, \mathcal{A})$ be a measurable space and $L$ a a bounded lattice. A non-additive measure on $A$ with values in $L$ is a function $m: \mathcal{A} \rightarrow L$ such that $m(\emptyset)=0, m(A)=1$ and $m(X) \leq m(Y)$ whenever $X \subseteq Y$.

A function $f: A \rightarrow L$ is said to be measurable if the sets $\{x: f(x) \leqslant a\}$ and $\{x: f(x) \geqslant a\}$ are elements of $\mathcal{A}$ for every $a \in L$. We will use $\{f \geqslant x\}$ to indicate the weak upper level set $\{t \in L: f(t) \geqslant x\}$.

We denote by $\mathcal{M}$ the set of all fuzzy measures on A with values in $L$ and by $\mathcal{F}$ the set of the measurable functions $f: A \rightarrow L$. Following [15] and [17] we give the following definition.

Definition 1. A mapping I: $\mathcal{F} \times \mathcal{M} \rightarrow L$ will be called a lattice-valued integral if the following properties are satisfied:

(i) for every $c \in L$ and $m \in \mathcal{M}, I(\mathbf{c}, m)=c$;

(ii) for each $m_{1}, m_{2}, \in \mathcal{M}$ with $m_{1} \leq m_{2}$ and $f_{1}, f_{2}, \in \mathcal{F}$ with $f_{1} \leq f_{2}$ we have $I\left(m_{1}, f_{1}\right) \leq I\left(m_{2}, f_{2}\right)$.

This general definition has to be completed by a variety of additional properties. In some cases we consider a lattice-valued integral as a function defined in $\mathcal{F}$. In order to obtain the additivity of the integral it is useful the concept of comonotonic functions. The concept of comonotonicity emerges quite naturally in many different fields such as aggregation theory, decision theory, finance and actuarial sciences (see [22] ) and comonotonicity was already used under different names by many authors. We refer to Denneberg [8] for the definition as well as for different characterizations of comonotonicity. In [22] several multivariate extensions of the classical definition have been studied. In this paper we propose a generalization of the notion of comonotonicity to the case of lattice-valued functions.

If $\mathrm{A}$ is a non empty set and $L$ is a lattice two function $f, g: A \rightarrow L$ are said to be comonotone if for every $x \in L$

$$
\text { either }\{f \geqslant x\} \supseteq\{g \geqslant x\} \text { or }\{g \geqslant x\} \supseteq\{f \geqslant x\}
$$


We consider now some of the properties that a lattice-valued integral $I: \mathcal{F} \rightarrow L$ may or may not satisfy:

(i) (homogeneity) $I(f \wedge \mathbf{c})=I(f) \wedge c$ for every $c \in L$ and for every $f \in \mathcal{F}$;

(ii) (invariance): $I(\gamma \circ f)=(\gamma \circ I)(f)$ for every continuous mapping $\gamma: L \rightarrow L$ and for every $f \in \mathcal{F}$;

(iii) (comonotone maxitivity): $I(f \wedge g)=I(f) \wedge I(g)$ if $f, g$ are comonotone elements of $\mathcal{F}$.

It is easy to prove that an invariant integral is homogeneous.

\section{Quantiles and Sugeno integrals in complete lattices}

Here we provide a definition and characterization of quantiles for lattice-valued operators. In this section we assume that $L$ is a completely distributive lattice and that $\mathcal{A}=2^{A}$.

Definition 2. If $\alpha$ is an element of $L$ the lattice-valued quantile of level $\alpha$ is the functional $Q_{\alpha}: \mathcal{F} \times \mathcal{M} \rightarrow L$ such that

$$
Q_{\alpha}(f, m)=\bigvee\{x: m(\{f \geqslant x\}) \geqslant \alpha\} .
$$

It can be proved that this definition extends the well known definition of quantile for real-valued functions(see [5]).

Say that a collection of sets $\mathcal{U} \subseteq 2^{A}$ is an upper set if $X \in \mathcal{U}$ and $X \subset Y$ implies that $Y \in \mathcal{U}$. Then we can prove the following result.

Proposition 1. A lattice-valued integral $I: \mathcal{F} \rightarrow L$ is a lattice-valued quantile with respect to a non-additive measure $m: \mathcal{A} \rightarrow L$ if and only if there exists a upper set $\mathcal{U}$ such that

$$
I(f)=\bigvee_{X \in \mathcal{U}} \bigwedge_{x \in X} f(x)
$$

or if and only if there exists a upper set $\mathcal{U}$ such that

$$
I(f)=\bigwedge_{X \in \mathcal{U}} \bigvee_{x \in X} f(x)
$$

Proof. If $I$ is a lattice-valued quantile we can consider the upper set $\mathcal{U}=\{X \in$ $\left.2^{A}: m(X) \geq \alpha\right\}$ and we can get

$$
I(f)=\bigvee_{X \in \mathcal{U}} \bigwedge_{x \in X} f(x)
$$

By Theorem 5 in [4] we have that

$$
\bigvee_{X \in \mathcal{U}} \bigwedge_{x \in X} f(x)=\bigwedge_{X \in \mathcal{V}} \bigvee_{x \in X} f(x)
$$


where $\mathcal{V}=\{Y \subseteq A: X \cap Y \neq \emptyset$ for every $X \in \mathcal{U}\}$. It is easy to prove that $\mathcal{V}$ is an upper set being $\mathcal{U}$ an upper set.

Conversely given the upper set $\mathcal{U}$ if we define a non-additive measure $m: \mathcal{A} \rightarrow L$ such that $m(X)=1$ if $X \in \mathcal{U}$ and $m(X)=0$ if $X \notin \mathcal{U} I(f)$ is a a lattice-valued quantile with respect to the non-additive measure $m$.

We can immediately prove that a lattice-valued quantile $Q_{\alpha}: \mathcal{F} \times \mathcal{M} \rightarrow L$ is an integral. The following proposition characterizes quantiles as functionals $Q_{\alpha}: \mathcal{F} \rightarrow L$ in completely distributive lattices.

Proposition 2. A lattice-valued quantile is an invariant and comonotone maxitive functional such that for every $X \subseteq A$ either $Q_{\alpha}(\mathbf{X})=\mathbf{1}$ or $Q_{\alpha}(\mathbf{X})=\mathbf{0}$. If $I: \mathcal{F} \times \mathcal{M} \rightarrow L$ is an integral such that either $I(\mathbf{X})=1$ or $I(\mathbf{X})=0$ then $I$ is a lattice-valued quantile if and only if $I$ is invariant.

Proof. The result follows easily from Theorem 3 in [5], we have only to prove that a lattice-valued quantiles is comonotone maxitive.

If $f, g: A \rightarrow L$ are two comonotone functions, then $\{(f \vee g) \geqslant x\}=\{f \geqslant$ $x\}) \cup\{g \geqslant x\}$ is equal to $\{f \geqslant x\}$ or to $\{g \geqslant x\}$. Then we can prove that $m(\{(f \vee g) \geqslant x\})=m\left(\{(f \geqslant x\}) \vee m\left(\{(g \geqslant x\})\right.\right.$. Hence it follows that $Q_{\alpha}(f \vee g)=$ $\bigvee\{x: m(\{(f \vee g) \geqslant x\}) \geqslant \alpha\}=Q_{\alpha}(f) \vee Q_{\alpha}(g)$.

We are interested in a class of integral functionals defined on a complete lattice. Following the approach in [13] we consider the functionals $S_{l}, S_{u}$ defined by :

$$
\begin{aligned}
S_{l}(m, f) & =\bigvee_{x \in L}(x \wedge m(\{f \geqslant x\}) \quad \text { and } \\
S_{u}(m, f) & =\bigwedge_{x \in L}(x \vee m(\{x: f(x)) \not \leq x\}) .
\end{aligned}
$$

If $L$ is a completely distributive lattice $S=S_{l}=S_{u}$ and the functional $S$ extends Sugeno integral to an ordinal framework and so is called the lattice-valued Sugeno integral of $f$ with respect to $m$. The following proposition provides an axiomatic representation of this functional.

Proposition 3. A lattice-valued integral $I: \mathcal{F} \times \mathcal{M} \rightarrow L$ is a lattice-valued Sugeno integral that is

$$
I(m, F)=\bigvee_{x \in L}(x \wedge m(\{f \geqslant x\})
$$

if and only if it is homogeneous and comonotone maxitive.

Proof. It is straightforward to show that a lattice-valued Sugeno integral is monotone and homogeneous. Using the properties of comonotone functions as in the proof of Proposition 2 we can prove that the functional $S(f)$ is comonotone maxitive. Then we consider a comonotone maxitive and homogeneous integral 
$I=I(f)$. In [13] it is proved that this functional is a Sugeno integral if when $f, g: A \rightarrow L$ are two functions such that $f \leq g$ and $a \in L$ we have that

$$
I(f \vee(a \wedge g))=I(f) \vee(a \wedge I(g)) .
$$

If $a \geq x$ then $\{f \geqslant x\} \supseteq\{g \wedge a \geqslant x\}$ and if $a \nsupseteq x$ we have that $\{g \wedge a \geqslant x\}=\emptyset$ and then the functions $f, g \wedge a$ are comonotone. Hence we have $I(f \vee(a \wedge g))=$ $I(f) \vee I(a \wedge g)$ and then since $I$ is homogeneous and $I(a \wedge g))=a \wedge I(g)$ an so the claim is proved.

Here we characterize quantiles as a subclass of the Sugeno integrals.

Proposition 4. A lattice-valued Sugeno integral $S: \mathcal{F} \rightarrow L$ is a lattice-valued quantile if and only if there exists a $\{0,1\}$-valued non-additive measure $m \in \mathcal{M}$ such that $I(f)=S(f, m)$.

Proof. If $S$ is a lattice-valued quantile of level $\alpha$ with respect to the non additive measure $m \in \mathcal{M}$ can consider the non-additive measure $m^{*} \in \mathcal{M}$ such that $m^{*}(X)=1$ if $m^{*}(X) \geq \alpha$ and $m^{*}(X)=0$ otherwise. Conversely if $S$ is latticevalued quantile of level $\alpha$ with respect to the $\{0,1\}$-valued non-additive measure $m \in \mathcal{M}, S$ is a a lattice-valued quantile of level $\alpha$ for every $\alpha \neq 0$.

We can also prove that the subclass of quantiles generates the class of Sugeno integrals.

Proposition 5. If $S: \mathcal{F} \times \mathcal{M} \rightarrow L$ is a lattice-valued Sugeno integral then for every $f \in \mathcal{F}$ and for every $m \in \mathcal{M}$ we have

$$
S(f, m)=\bigvee_{\alpha \in L}\left(Q_{\alpha}(f, m) \wedge \alpha\right)
$$

Proof. If $f$ is an element of $\mathcal{F}$ and $m$ is an element of $\mathcal{M}$ we have that the set $\left\{Q_{\alpha}(f, m): \alpha \in L\right\} \subseteq L$ and then $S(f, m)=\bigvee_{x \in L}(x \wedge m(\{f \geqslant x\}) \geq$ $\bigwedge_{\alpha \in L}\left(Q_{\alpha}(f, m) \wedge \alpha\right)$.

If $x \in L$ is such that $m(\{f \geqslant x\})=\alpha$ then $x \geq Q_{\alpha}$ hence we can prove that $S(f, m)=\bigvee_{x \in L}\left(x \wedge m(\{f \geqslant x\}) \leq \bigwedge_{\alpha \in L}\left(Q_{\alpha}(f, m) \wedge \alpha\right)\right.$.

\section{Real valued quantiles and integrals: some extensions}

Throughout this section $(A, \mathcal{A})$ be a measurable space (if $A$ is a finite set we usually assume that $\mathcal{A}=2^{A}$ ) and $L$ is the real interval $[0,1]$. It can be noticed that several type of integrals and in particular the Choquet integral were introduced considering real interval different from $[0,1]$, but they can be transformed into the $[0,1]$ framework.

If $\mathcal{M}$ is the class of $[0,1]$-valued non-additive measures defined on $A, \mathcal{F}$ is the class of $[0,1]$-valued measurable functions defined on $A$ and $f \in \mathcal{F}$ the Choquet integral is a mapping $C: \mathcal{F} \times \mathcal{M} \rightarrow[0,1]$ defined by

$$
C(f, m)=\int f d m=\int_{0}^{1} m(\{f \geqslant x\}) d x .
$$


It is well known (see [8] and [9] for example) that Choquet integral is a comonotone linear functional i.e. $I(a f+b g)=I(a f)+I(b g)$ if $f, g$ are comonotone elements of $\mathcal{F}$ and $0 \leq a, b \leq 1$.

Moreover it can be proved that every integral functional $I: \mathcal{F} \times \mathcal{M} \rightarrow[0,1]$ that is comonotone linear is a Choquet integral (see [8] or [9]).

The following proposition proves that, as in the case of Sugeno integrals, the quantiles functionals are Choquet integrals and that the subclass of quantiles generates the class of Choquet integrals.

Proposition 6. The Choquet integral $I: \mathcal{F} \rightarrow[0,1]$ is a quantile if and only if there exists a $\{0,1\}$-valued non-additive measure $m \in \mathcal{M}$ such that $I(f)=$ $C(f, m)$.

If $C: \mathcal{F} \times \mathcal{M} \rightarrow L$ is a $[0,1]$-valued Choquet integral then for every $f \in \mathcal{F}$ and for every $m \in \mathcal{M}$ we have

$$
C(f, m)=\int_{0}^{1} Q_{\alpha}(f, m) d \alpha .
$$

Proof. If $I$ is a lattice-valued quantile of level $\alpha$ with respect to the non additive measure $m \in \mathcal{M}$ we consider the non-additive measure $m^{*} \in \mathcal{M}$ such that $m^{*}(X)=1$ if $m^{*}(X) \geq \alpha$ and $m^{*}(X)=0$ otherwise.

Then it is easy to prove that if $q=Q_{\alpha}(f, m)$

$$
C\left(f, m^{*}\right)=\int f d m^{*}=\int_{0}^{q} m^{*}(\{f \geqslant x\}) d x=q .
$$

The equality $C(f, m)=\int_{0}^{1} Q_{\alpha}(f, m) d \alpha$ follows directly from proposition $1.4 \mathrm{in}$ $[8]$.

We have considered quantiles with respect to a (possibly non-additive) measure and not necessarily with respect to an endogenous probability. as in the classical case. Now we define quantiles with respect to a family of non-additive measures considering different attitude for low or high input values as in the definition of level-dependent integrals (see [18] and [12] ).

If $\left(m_{t}\right)$ is a family of elements of $\mathcal{M}$ and $t \in[0,1]$ we consider the generalized quantile of level $\alpha$, the generalized Sugeno integral and the generalized Choquet integral of a function $f \in \mathcal{F}$ as follows:

$$
\begin{gathered}
G Q_{\alpha}(f)=\bigvee\left\{x: m_{\alpha}(\{f \geqslant x\}) \geqslant \alpha\right\} ; \\
G S(f)=\bigvee_{\alpha \in L}\left(Q_{\alpha}\left(f, m_{\alpha}\right) \wedge \alpha\right) \text { and } \\
G C(f)=\int_{0}^{1} Q_{\alpha}\left(f, m_{\alpha}\right) d \alpha .
\end{gathered}
$$

The proposed generalized integrals satisfy some minimal properties. 
Proposition 7. The generalized Sugeno integral and Choquet integral are monotone functionals. The generalized Sugeno integral is comonotone maxitive while the generalized Choquet integral is comonotone additive.

Proof. Let us check comonotone maxitivity (additivity) of generalized Sugeno (Choquet) integral. If $f, g \in \mathcal{F}$ are comonotone functions then for every $\alpha \in$ then $G Q_{\alpha}(f \vee g)=G Q_{\alpha}(f) \vee G Q_{\alpha}(g)$ and $G Q_{\alpha}(f+g)=G Q_{\alpha}(f)+G Q_{\alpha}(g)$ and then the two properties are easily proved.

The integrals with respect to non-additive measures proved useful in many areas of decision theory. As it is well known in decision under uncertainty it leads to a generalization of expected utility. In this framework the aggregation functionals defined above introduces a a multiple quantiles model for a decision-making process under ambiguity in which a decision-maker is supposed to consider a rank among outcomes. Following the approach in [3] the generalized functionals are able to represent asymmetric attitude on extreme events (unexpected gains or unusual losses) and a rational prudence on ordinary events.

\section{Application to citation analysis}

Assessment of the quality of research has become increasingly necessary in recent years and many different indicators have been studied. We consider the approach in which the quality of a a research output is measured by citation analysis. Among the numerous bibliometric indices that have been used to evaluate the scientific production of a researcher or a scientific journal, a very popular index is the $h$-index which take into account the quality of the output of a scientist represented by the number of citations per paper and the impact represented by the number of paper ([14]). This index is relatively recent but the scientific community has shown a considerable interest for this indicator. The $h$-index of a researcher is the maximum number $h$ of papers of the considered scientist having at least $h$ citations each. The $h$-index is a particular case of Sugeno integral (see $[20])$ and obviously it is an aggregation operator.

Many recent papers generalize this approach and use aggregation functions in the analysis of citation data (see [21]). . Prospect Theory in an am We introduce a generalization of this index to quantify an individual's scientific work, considering in particular excellent papers.

Let $\mathbb{I}=[0,+\infty)$ denote the interval of nonnegative real numbers. We consider also a finite index set $N=\{1, \ldots, n\}$ and a non-additive measure $m: 2^{N} \rightarrow \mathbb{I}$.

Let an author's output be characterized by a set of $N$ publications and, for each publication, the number of citations of that paper. We take here the number of citations as given and we consider the number of citations of a paper as a measure of the paper's quality. We represent a researcher by a function $f: N \rightarrow \mathbb{I}$ where $f(i)$ is the number of citations of the $i$ th publication and we assume $f(i)=0$ if the considered author has less than $i$ publications. 
An impact index is an aggregation function $I: \mathbb{I}^{N} \rightarrow \mathbb{I}$.

If the measure $m$ is the counting measure the impact index defined by

$$
F(f)=S(m, f)=\int_{0}^{1} Q_{\alpha}(f, m) d \alpha
$$

is the h-index. The $h$-index identifies the most cited papers and it is insensitive to low cited papers. However this index don't identify researcher that have a moderate level of production but a very high impact. In some cases, to have large $h$-index is to have many good papers while a scientist with few papers with a high number of citations per paper in general has not a high $h$-index. For a discussion of this and other weakness of the $h$-index we refer to [1]. Now where are considered also a number of $h$-type indices proposed in the literature.

If $\left(m_{t}\right)$ is a family of non-additive measures on $N$ and $t \in \mathbb{I}$ we may consider the impact index defined by

$$
\begin{aligned}
G C(f) & =\int_{0}^{+\infty} Q_{\alpha}\left(f, m_{\alpha}\right) d \alpha \text { where } \\
Q_{\alpha}(f) & =\bigvee_{\alpha \in \mathbb{I}}\left\{x: m_{\alpha}(\{f \geqslant x\}) \geqslant \alpha\right\} .
\end{aligned}
$$

In this case we obtain a more flexible index which takes into account the degree of importance of a given level of citations. The proposed indices can be considered as generalized $h$-type indices. For a more detailed discussion on a number of generalized $h$-type indices proposed in the literature see the paper [1].

\section{Concluding remarks}

We introduced a unified qualitative framework for studying non-additive measures and integration theory based on the notion of quantile.

The focus has been on aggregation functionals defined on lattices. In particular we have introduced integral-based aggregation functionals defined on completely distributive lattices.

For real-valued functions we introduce some functionals that generalize Sugeno and Choquet integrals and a further research direction is that of an axiomatic characterization of the considered aggregation functionals.

It is important to note that the proposed definition of generalized Sugeno integral can be easily extended to an ordinal framework. We have shown with an example that the proposed generalized integrals can be applied in real problems.

\section{References}

1. Alonso S., Cabrerizo F.J., Herrera-Viedmac E., Herrera F. H., H-index: A Review Focused in its Variants, Computation and Standardization for Different Scientific Fields, Journal of Informetrics, 3, 273-1289, (2009). 
2. Ban A., Fechete I., Componentwise decomposition of some lattice-valued fuzzy integrals, Information Sciences,177, 1430-1440, (2007).

3. Basili M., Chateauneuf A., Extreme events and entropy: a multiple quantile utility model, International Journal of Approximate Reasoning, 52, 1095-1102, (2011).

4. Cardin M., Couceiro M., Invariant functionals on completely distributive lattices, Fuzzy Sets and Systems, 167, 45-56, (2011).

5. Cardin M., Couceiro M., An Ordinal Approach to Risk Measurement in C.Perna, M.Sibillo eds, Mathematical and Statistical Methods for Actuarial Science and Finance, Springer-Verlag, 79-86, (2012).

6. Chambers C., Ordinal Aggregation and Quantiles, Journal of Economic Theory, 137, 416-43, (2007).

7. Davey B.A, Priestley H.A., Introduction to Lattices and Order, Cambridge University Press, New York, (2002).

8. Denneberg D.,Non-additive measure and integral, Kluwer Academic Publisher, Dordrecht, (1994).

9. Grabisch M. .Marichal J.L, Mesiar R., Pap E., Aggregation Functions, Encyclopedia of Mathematics and its Applications. Cambridge University Press, Cambridge, (2009).

10. Grätzer G., General Lattice Theory, Birkhäuser Verlag, Berlin, (2003).

11. Gierz G., Hofmann K.H., Keimel K., Lawson J.D, Mislove M.D., Scott D.S.. Continuous Lattices and Domains, Cambridge University Press, Cambridge, (2003).

12. Giove S., Greco S., Matarazzo B., The Choquet Integral with respect to level dependent capacity, Fuzzy Sets and Systems, 175, 1-35, (2011).

13. Greco G.H., Fuzzy Integral and Fuzzy Measures with Their Values in Complete Lattices, Journal of Mathematical Analysis and Applications 126, 594-603, (1987).

14. Hirsch J. E., An index to quantify an indivividual's scientific research output, Proceedings of the National Academy of Sciences of USA, 102, 16569-16572 (2005).

15. Klement E.P., Mesiar R., and Pap E., A Universal Integral Proceedings EUSFLAT 2007, Ostrava, vol. I, 253-256, ( 2007).

16. Mesiar R., Fuzzy measures and integrals, Fuzzy Sets and Systems,156, 365-370, (2005).

17. Mesiar R., Fuzzy integrals and linearity, International Journal of Approximate Reasoning, 4 352-358, (2008).

18. Mesiar R., Mesiarová Zemánková A., Ahmad K., Level-dependent Sugeno Integral, IEE Transactions on Fuzzy Systems, 171, 167-172 (2009).

19. Murofushi T., Sugeno M., Some quantities represented by the Choquet integral, Fuzzy Sets and Systems, 5, 229-235, (1993).

20. Narukawa Y., Torra:V., The h-Index and the Number of Citations: Two Fuzzy Integrals, IEE Transactions on Fuzzy Systems, 16, 795-797, (2008).

21. Narukawa Y., Torra:V., Multidimensional generalized fuzzy integral, Fuzzy Sets and Systems 160, 802-815, (2009).

22. Puccetti G., Scarsini M. Multivariate comonotonicity, Journal of Multivariate Analysis 101, 291-304 (2010). 